\title{
Vorsicht bei der Wahl des Praxisvertreters
}

\author{
Der Beruf des Facharztes ist ein "freier Beruf". So verspricht es die \\ Bundesärzteordnung. Tatsächlich ist der Facharzt in seinem Beruf \\ von vielfältigen Vorgaben und Restriktionen „umzingelt“. Dies gilt \\ nicht zuletzt für Praxisvertretungen bei Krankheit und Urlaub.
}

Will ein Facharzt im Falle von Urlaub, Fortbildung, Krankheit etc. seine Praxis nicht schließen, muss er für einen fachlich qualifizierten Vertreter während seiner Abwesenheit sorgen, der stellvertretend für ihn als Inhaber des Unternehmens handelt. Da lauern ein paar Fallstricke: $\mathrm{Zu}$ beachten ist die Gefahr von Honorarrückforderungen durch die $\mathrm{KV}$, sofern im Nachhinein eine unwirksame Vertretung nachgewiesen wird. Für die Abrechnung von Leistungen, die eine besondere Qualifikation erfordern, muss auch der Praxisvertreter über einen entsprechenden Qualifikationsnachweis verfügen und gegebenenfalls vorlegen. Schließlich empfiehlt es sich zur "Regressprophylaxe“ auch den Praxisvertreter auf die Beachtung des Wirtschaftlichkeitsgebots und sonstigen Vorgaben in einer Kassenpraxis einzuschwören.

\section{Vertrauen ist gut, Kontrolle ist besser}

Der zentrale Punkt: Der Facharzt muss sich davon überzeugen, dass der gewählte Praxisvertreter tatsächlich fachlich geeignet ist, die Vertretung zuverlässig zu übernehmen. Grundsätzlich muss dieser eine abgeschlossene Weiterbildung in demselben Fachgebiet vorweisen können. Vertragsärzte dürfen sich nur durch andere Vertragsärzte oder durch Ärzte vertreten lassen, die die Voraussetzungen für die Eintragung in das Arztregister erfüllen (Approbation als Arzt und die Facharztanerkennung für das zu vertretende Fachgebiet). Praxisvertretungen sind auch zulässig, wenn der Kollege zwar nicht über eine Approbation, aber über eine Berufserlaubnis verfügt, die ihm die selbstständige Tätigkeit als Praxisvertreter erlaubt. Die Praxis in eigener Abwesenheit führen kann auch ein Krankenhausarzt. Eine Altersbegrenzung für die Praxisvertretung gibt es nicht.

Vertrauen ist gut, Kontrolle besser: Der Facharzt ist gut beraten, sich vor dem Vertragsabschluss die Originalexemplare

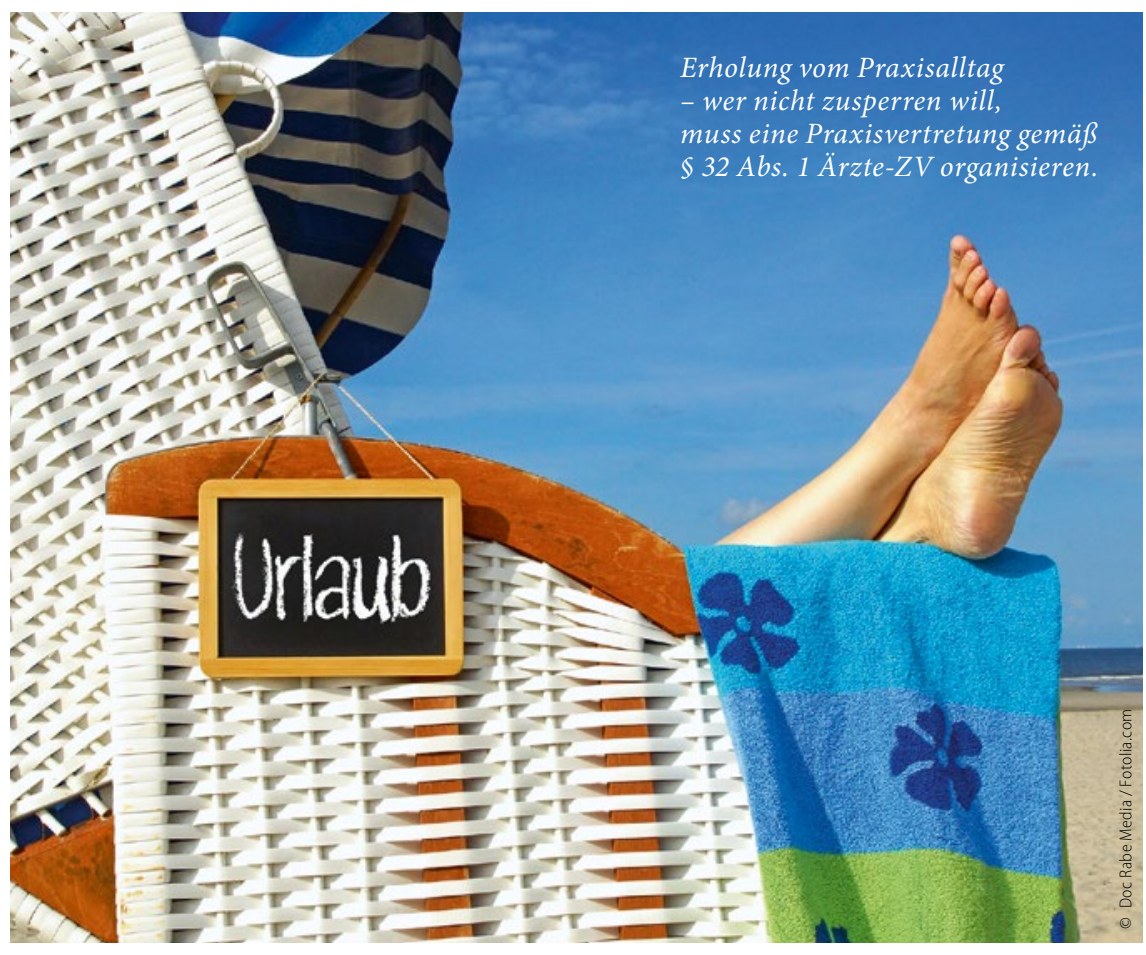

oder beglaubigten Kopien der Approbations- und der Facharzturkunde anzusehen. $\mathrm{Zu}$ empfehlen ist auf jeden Fall ein wasserdichter schriftlicher Vertrag. Nicht zu vergessen sind auch die möglicherweise existenzgefährdenden Risiken bei der Haftung für Behandlungsfehler des Praxisvertreters, der den Arztvertrag mit dem Patienten ja im Namen des Praxisinhabers abschließt. Daraus ergibt sich, dass der Vertreter gegenüber dem Patienten für Behandlungsfehler aus eigener unerlaubter Handlung haftet, während der Praxisinhaber für alle weiteren Fehlleistungen in der Haftung steht. Um unangenehme Überraschungen zu vermeiden, sollte sich der Facharzt vergewissern, dass seine Haftpflichtversicherung auch Behandlungsfehler seines Vertreters umfasst. Der Praxisvertreter seinerseits muss für eine Berufshaftpflichtversicherung für die eigene selbstständige Tätigkeit sorgen.

Norbert Fischer
Regelung der Praxisvertretung nach $\S 32$ Abs. 1 der Ärzte-ZV

Der Vertragsarzt hat die vertragsärztliche Tätigkeit persönlich in freier Praxis auszuüben. Bei Krankheit, Urlaub oder Teilnahme an ärztlicher Fortbildung oder an einer Wehrübung kann er sich innerhalb von zwölf Monaten bis zur Dauer von drei Monaten vertreten lassen. Eine Vertragsärztin kann sich in unmittelbarem zeitlichen Zusammenhang mit einer Entbindung bis zu einer Dauer von zwölf Monaten vertreten lassen. Dauert die Vertretung länger als eine Woche, so ist sie der KV mitzuteilen. Der Vertragsarzt darf sich grundsätzlich nur durch einen anderen Vertragsarzt oder durch einen Arzt, der die Voraussetzungen des $\S 3$ Abs. 2 (Approbation und Weiterbildung) erfüllt, vertreten lassen. Überschreitet innerhalb von zwölf Monaten die Dauer der Vertretung einen Monat, kann die KV beim Vertragsarzt oder Vertreter überprüfen, ob der Vertreter die Voraussetzungen nach Satz 5 erfüllt und keine Ungeeignetheit nach $\S 21$ vorliegt. 